\title{
Ferroelectric Phase Transition in DC Electric Field in $\left[\mathrm{NH}_{2}\left(\mathrm{CH}_{3}\right)_{2}\right]_{3} \mathrm{Sb}_{2} \mathrm{Cl}_{9}$ Crystals
}

\author{
J. DZIEDZic AND J. Mróz
}

Institute of Physics, Wrocław University of Technology

Wybrzeże Wyspiańskiego 27, 50-370 Wrocław, Poland

(Received February 21, 2007; revised version April 16, 2007)

Dielectric investigations of ferro-paraelectric phase transition under influence of bias pooling DC electric field ranging from 0 to $500 \mathrm{kV} / \mathrm{m}$ were performed for $\left[\mathrm{NH}_{2}\left(\mathrm{CH}_{3}\right)_{2}\right]_{3} \mathrm{Sb}_{2} \mathrm{Cl}_{9}$. The linear shift of $T_{\mathrm{c}}$ towards higher temperatures was observed. The $A_{0}$ and $B$ coefficients from the Landau state equation are determined.

PACS numbers: $64.70 . \mathrm{Kb}$

\section{Introduction}

Alkylammonium halogenoantimonates(III) with the general formula $\mathrm{R}_{3} \mathrm{Sb}_{2} \mathrm{X}_{9}$ (where $\mathrm{R}=\left(\mathrm{CH}_{3}\right)_{n} \mathrm{NH}_{4-n}, \mathrm{X}=\mathrm{Cl}, \mathrm{Br}, \mathrm{I}$ ) have been extensively investigated from the viewpoint of ferroelectricity. The reorientations of the alkylammonium cations are found to play a dominant role in the mechanism of numerous "order-disorder" phase transitions [1-3]. $\left[\mathrm{NH}_{2}\left(\mathrm{CH}_{3}\right)_{2}\right]_{3} \mathrm{Sb}_{2} \mathrm{Cl}_{9}$ (DMACA) crystallizes at room temperature (RT) in the monoclinic symmetry with the space group $P 2_{1} / c$ [4]. The RT structure consists of two-dimensional layer structure of $\left(\mathrm{Sb}_{2} \mathrm{Cl}_{9}^{3-}\right)_{n}$ anions and two sets of nonequivalent dimethylammonium cations (DMA). One type of DMA cations located inside the polyanionic cavities is disordered, whereas the second type of cations is placed between the layers. The former DMA cations are believed to contribute to the mechanism of phase transition. DMACA undergoes continuous ferroelectric phase transition from $P 2_{1} / c$ to $P c$ at $242 \mathrm{~K}$ [5-7]. DMACA possesses also ferroelastic properties at RT and the hypothetical paraelastic phase $3 m$ was proposed [8]. The dielectric dispersion studies in the microwave frequency region revealed the critical slowing down of the macroscopic relaxation characteristic of the second-order phase transition [9].

The purpose of the present work is to study both the influence of the pooling electric field on the dielectric permittivity and character of the phase transition. 


\section{Experimental}

Single crystals of $\left[\mathrm{NH}_{2}\left(\mathrm{CH}_{3}\right)_{2}\right]_{3}\left[\mathrm{Sb}_{2} \mathrm{Cl}_{9}\right]$ was precipitated from the stoichiometric aqueous solution of $\mathrm{Sb}_{2} \mathrm{O}_{3}$ and $\mathrm{NH}_{2}\left(\mathrm{CH}_{3}\right)_{2} \mathrm{Cl}$ at a high excess of $\mathrm{HCl}$ and purified by a recrystallization. A single crystal was grown by an evaporation of the solution at constant room temperature. The sample with dimensions $5 \times 5 \times 1 \mathrm{~mm}^{3}$ of DMACA was investigated. The surfaces perpendicular to the ferroelectric $a$-axis were provided with silver paste electrodes. The real part $\left(\varepsilon_{a}\right)$ of the complex electric permittivity, $\varepsilon^{*}$, was measured using an automatic RLC meter at $1 \mathrm{kHz}$ frequency in AC field amplitude equal to $500 \mathrm{~V} / \mathrm{m}$. The temperature of the sample was changed with a cooling rate $0.1 \mathrm{~K} / \mathrm{min}$.

\section{Results and discussion}

The electric permittivity vs. temperature, $\varepsilon_{a}^{\prime}(T)$, at various values of constant DC electric field is shown in Fig. 1. The electric field $(E)$ lowers the maximum of the electric permittivity, $\varepsilon_{\mathrm{m}}$, and it shifts the temperature $T_{\mathrm{m}}$, at which that maximum occurs, according to the following relation:

$$
T_{\mathrm{c}}-T_{\mathrm{m}}=\sqrt[3]{\frac{3 B}{A_{0}}} E^{2 / 3}
$$

where $T_{\mathrm{c}}-T_{\mathrm{m}}=\Delta T, T_{\mathrm{c}}-$ Curie-Weiss temperature.

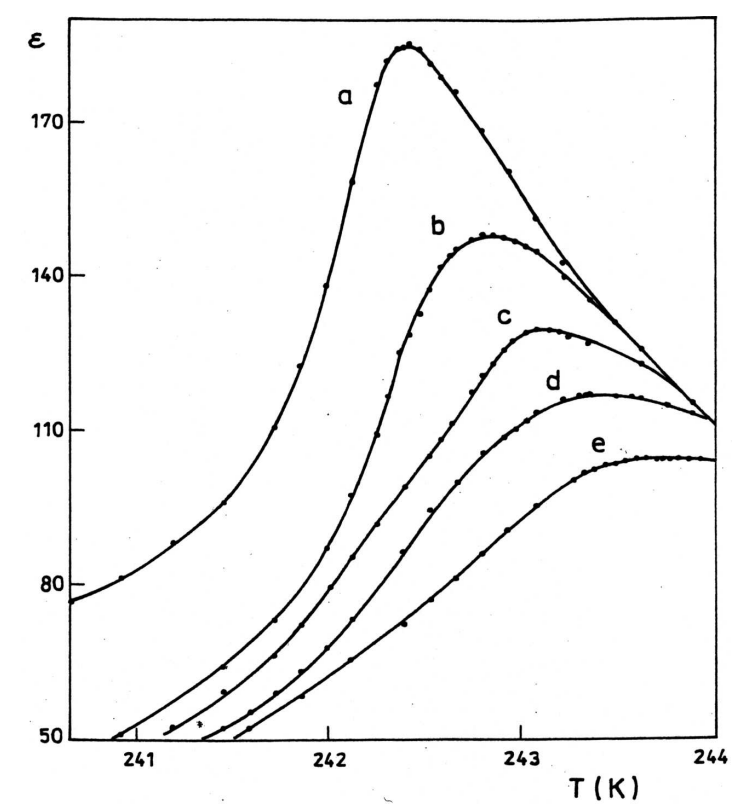

Fig. 1. Temperature dependences of the electric permittivity on cooling around the phase transition temperature at various external electric fields: $a-0 \mathrm{~V} / \mathrm{m}, b-$ $100 \mathrm{kV} / \mathrm{m}, c-200 \mathrm{kV} / \mathrm{m}, d-300 \mathrm{kV} / \mathrm{m}, e-500 \mathrm{kV} / \mathrm{m}$. 


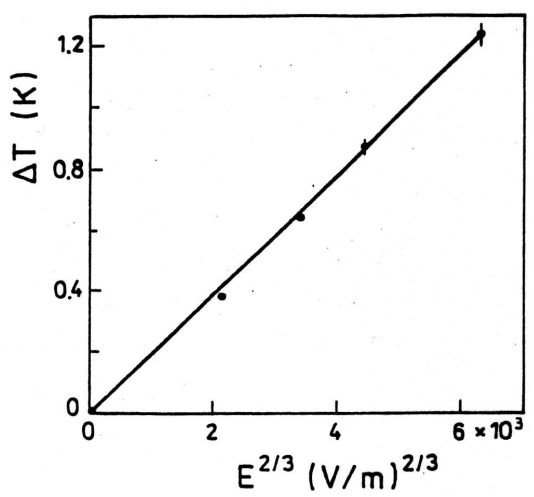

Fig. 2. The dependence of $\Delta T$, at which maximum of $\varepsilon$ occurs, as a function of $2 / 3$ power of DC electric field value.

In Fig. 2 the dependence of $\Delta T$ on $E^{2 / 3}$ is presented.

Using a simple relation between the Curie-Weiss constant $\left(C_{\mathrm{CW}}=400 \mathrm{~K}\right)$ and $A_{0}$ coefficient

$$
C_{\mathrm{CW}}=\frac{1}{\varepsilon A_{0}},
$$

the last coefficient was calculated to be $1.41 \times 10^{8} \mathrm{~V} \mathrm{~m} /(\mathrm{C} \mathrm{K})$. The $B$ coefficient for the paraelectric phase calculated from Eq. (1) is equal to $7 \times 10^{12} \mathrm{~V} \mathrm{~m}^{5} \mathrm{C}^{-3}$.

It is interesting that the value of the $B$ coefficient for DMACA crystals estimated for the paraelectric phase is comparable to those found for the closely related ferroelectric crystals like $\left(\mathrm{CH}_{3} \mathrm{NH}_{3}\right)_{5} \mathrm{Bi}_{2} \mathrm{Br}_{11}\left(3.6 \times 10^{12} \mathrm{~V} \mathrm{~m}^{5} \mathrm{C}^{-3}\right)[10]$ and $\left(\mathrm{CH}_{3} \mathrm{NH}_{3}\right)_{5} \mathrm{Bi}_{2} \mathrm{Cl}_{11}\left(7.2 \times 10^{12} \mathrm{~V} \mathrm{~m}^{5} \mathrm{C}^{-3}\right)[11]$.

It seems that these coefficients are frequently encountered for ferroelectric crystals which exhibit continuous "order-disorder" phase transition.

\section{References}

[1] R. Jakubas, L. Sobczyk, Phase Transit. 20, 163 (1990).

[2] V. Varma, R. Bhattacharjee, H.N. Vasan, C.N.R. Rao, Spectrochim. Acta A 48, 1631 (1992).

[3] H. Ishihara, K. Watanabe, A. Iwata, K. Yamada, Y. Kinishita, T. Okuda, V.G. Krishnan, S. Dou, A. Weiss, Z. Naturforsch. A 47, 65 (1992).

[4] M. Gdaniec, Z. Kosturkiewicz, R. Jakubas, L. Sobczyk, Ferroelectrics 77, 31 (1988).

[5] J. Zaleski, A. Pietraszko, Acta Crystallogr. B 52, 287 (1996).

[6] R. Jakubas, Solid State Commun. 60, 389 (1986).

[7] A. Miniewicz, R. Jakubas, Solid State Commun. 63, 933 (1987).

[8] B. Kosturek, J. Przesławski, R. Jakubas, Solid State Commun. 75, 673 (1990).

[9] G. Bator, R. Jakubas, Phys. Status Solidi A 147, 591 (1995). 
[10] R. Cach, R. Jakubas, Ferroelectrics 110, 271 (1990).

[11] R. Cach, R. Jakubas, Ferroelectrics 108, 121 (1990). 